\title{
STRONGYLOIDES STERCOLARIS EN LAVADO BRONCOALVEOLAR
}

Fernando Polo MD*, Karen Shirley Avellaneda**, Paola Andrea Garnica**, Jennifer Alejandra López**, Andrés Gómez*** Rafael Parra-Medina****

\section{Resumen}

Las estrongiloidiosis es una infección parasitaria frecuente en zonas tropicales y subtropicales. Suele ser asintomática y limitarse al intestino. Sin embargo, pueden darse casos de infección extraintestinal diseminada y potencialmente fatales en pacientes inmunocomprometidos. Se presenta el caso de una paciente diagnosticada con estrongiloidiosis mediante una muestra de lavado broncoalveolar procesada con los métodos de cytospin y citología convencional.

Palabras clave: Strongyloides stercolaris, lavado broncoalveolar (BAL), citología convencional, cytospin.

\section{STRONGYLOIDES STERCORALIS IN BRONCHOALVEOLAR LAVAGE SPECIMEN}

\section{Abstract}

Strongyloidiasis is a parasitic infection found especially in tropical and subtropical regions. It is usually an asymptomatic and limited disease of the gut. However, potentially fatal cases of disseminated hyperinfection in immunosuppressed patients can occur. We present the case of a female patient with strongyloidiasis in bronchoalveolar lavage specimen processed as cytospin preparations and conventional cytology.

Key words: Strongyloides stercolaris, bronchoalveolar lavage (BAL), conventional cytology, cytospin.

Fecha recibido: enero 30 de 2015 - Fecha aceptado: mayo 7 de 2015

* Coordinador del Servicio de Patología del Hospital Infantil Universitario de San José. Instructor Asistente, Fundación Universitaria de Ciencias de la Salud. Bogotá DC, Colombia.

** Estudiantes de citohistotecnólogía. Fundación Universitaria de Ciencias de la Salud. Bogotá DC. Colombia.
*** Estudiante IX semestre, Facultad de Medicina, Universidad de Ciencias Aplicadas U.D.C.A. Bogotá DC, Colombia.

**** Residente I de Patología. Fundación Universitaria de Ciencias de la Salud. Bogotá DC, Colombia. 


\section{Introducción}

La estrongiloidiosis es una parasitosis causada por el helminto nemátodo Strongyloides stercolaris, descrito por primera vez en $1876 .{ }^{1}$ Es una infección endémica en zonas tropicales y subtropicales como África, el sudeste asiático y América del sur. ${ }^{1}$ Se estima que entre 3 y 100 millones de personas alrededor del mundo están infectadas por este parasito. ${ }^{2}$ En Colombia la prevalencia de la enfermedad oscila entre 5 y $10 \%$. $^{3,4}$

S. stercolaris ingresa al organismo a través de la piel, viaja por vía sanguínea hacia el alvéolo pulmonar donde permanece cerca de un mes, asciende por la vía aérea hasta la faringe donde es deglutido y conducido al tracto gastrointestinal, colonizando el intestino delgado, en especial duodeno e íleon. La hembra adulta del helminto pone sus huevos en la mucosa intestinal, donde eclosionan saliendo las larvas que son expulsadas del organismo en las heces. ${ }^{1}$

\section{Reporte de caso}

Mujer en la quinta década de la vida que ingresa al servicio de urgencias por cuadro de dificultad respiratoria asociado con expectoración mucopurulenta y signos de respuesta inflamatoria sistémica. El cuadro hemático reporta leucocitos 23.100/ml, neutrófilos 18.900/ml hemogolbina $10.1 \mathrm{~g} / \mathrm{dL}$, hematocrito $32.5 \%$, volumen corpuscular medio $78.6 \mathrm{fL}$, hemoglobina corpuscular media $25.4 \mathrm{pg}$ y plaquetas $33.300 / \mathrm{ml}$. La paciente continuó con descenso de los niveles de hemoglobina hasta $3.5 \mathrm{~g} / \mathrm{dL}$, por lo cual requirió transfusión de glóbulos rojos empaquetados. Como antecedentes de importancia presentaba cistoadenocarcinoma ovárico derecho con metástasis a pelvis, asma, síndrome de Churg Strauss en estudio y colitis neutropénica. Se decide hospitalizar e iniciar manejo antibiótico con vancomicina por antecedente de neumonía complicada dos semanas antes. Continuó con signos de respuesta inflamatoria sistémica, el hemocultivo reportó crecimiento de Streptococcus viridans y se inició manejo antibiótico específico.

Se practicó ecocardiograma transtorácico, esofagogastroduodenoscopia y TAC abdominal sin alteraciones.
En la radiografía de tórax se observaron opacidades intersticiales generalizadas sin consolidaciones. La paciente entró en falla ventilatoria que requirió ventilación mecánica invasiva. Se realizó fibrobrobroncoscopia con lavado broncoalveolar analizado mediante citología convencional y cytospin, obteniendo hallazgos compatibles con infección por Strongyloides stercolaris (Figura 1). Después la paciente presentó paro cardiorrespiratorio que no respondió a maniobras de reanimación.

\section{Discusión}

La mayoría de los individuos infectados por S. stercolaris son asintomáticos o presentan manifestaciones inespecíficas intestinales (dolor abdominal, diarrea intermitente o persistente, o bien síntomas que pueden imitar una enfermedad inflamatoria intestinal), respiratorios (tos, disnea y bronquitis crónica) o dermatológicos (rash y prurito). ${ }^{5,6}$ No obstante, en sujetos afectados por síndrome de hiperinfección (en el que el tiempo y la magnitud de la infestación aumentan en forma desproporcionada) o por infección diseminada (definida por la presencia del parásito en órganos o sistemas diferentes a los propios de su ciclo vital habitual), los síntomas suelen ser más severos y tienen compromiso sistémico. Los factores de riesgo para adquirir la infección, la forma diseminada o el síndrome de hiperinfección por S. stercolaris son el tratamiento crónico con esteroides, alcoholismo, desnutrición, neoplasias malignas e infección por VIH o HTLV-1. ${ }^{5}$

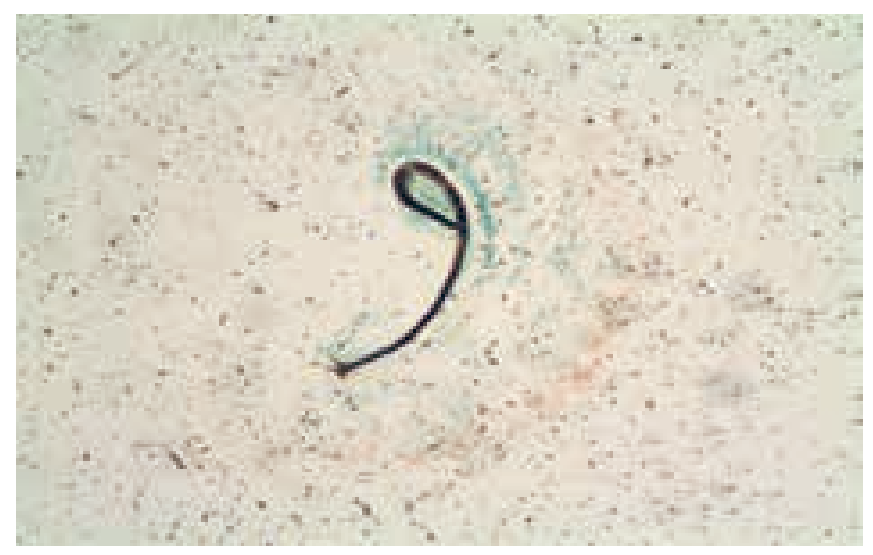

Figura I. Strongyloides stercolaris 10x. Muestra procesada por cytospin y coloreada con Gomory modificado. 
En el presente caso la paciente tenía una enfermedad maligna de base y manejo crónico de asma como factores de riesgo. Durante los últimos cuatro años presentó cuadros clínicos compatibles con estrongiloidiosis complicada, con lesiones cutáneas pruriginosas en cara, cuello, tórax, abdomen y miembros superiores acompañadas de tos productiva. Después tuvo varios ingresos hospitalarios por neumonías a repetición que cursaban con dolor pleurítico localizado en hemitórax derecho, anemia, pérdida de peso, dispepsia, diarrea, vómito e hiporexia.

Existen diversos métodos para establecer el diagnóstico de estrongiloidiosis. La detección microscópica del parásito en heces u otras muestras biológicas constituye el método más sencillo y económico, con una sensibilidad de $75.9 \%$ en la primera muestra y aumenta a $92 \%$ cuando se hacen tres exámenes. ${ }^{7}$ Métodos más recientes como la detección sérica de anticuerpos con sensibilidad de $65-100 \%$ y especificidad de $75-96 \%$ tienen el inconveniente de no poder diferenciar la estrongiloidiosis de otras infecciones parasitarias. ${ }^{2}$ Las técnicas de alta sensibilidad y especificidad como la reacción en cadena de polimerasa (RCP) con sensibilidad de $73-100 \%$ y especificidad de $96-100 \%$, tienen la desventaja de ser costosas, dispendiosas y presentar reactividad cruzada con otras parasitosis causadas por nemátodos. ${ }^{7,8} \mathrm{El}$ sistema de inmunoprecipitación por luciferasa (LIPS), uno de los más recientes para el diagnóstico de estrongiloidiosis, posee una sensibilidad de $97 \%$ y especificidad de $100 \%$, pero aún no se encuentra disponible en los laboratorios para diagnóstico de rutina. ${ }^{8}$

Por lo anterior, la visualización microscópica directa del parásito en sus diferentes estadios de desarrollo constituye el método más rápido, accesible y específico para el diagnóstico. La detección microscópica de larvas en las heces puede realizarse a través de los siguientes métodos: frotis simple directo (sensibilidad 0-52\%), concentración con formalina-éter (sensibilidad 13-55\%), cultivo con papel filtro de Harada-Mori (sensibilidad 13-55\%), técnica de Baerman o migración larvaria de heces a agua (sensibilidad 75\%) y cultivo en agar plata (sensibilidad 78-100\%). ${ }^{9}$
No obstante, para el caso específico de síndromes de hiperinfección o infecciones diseminadas causadas por S. stercolaris el examen citológico e histológico de los tejidos afectados constituye el método diagnóstico con mejor sensibilidad y especificidad. ${ }^{8,9,10}$ El lavado broncoalveolar que consiste en instalar el fibrobroncoscopio en un bronquio subsegmentario para realizar sucesivos lavados con solución salina y recoger el producto obteniendo material celular y acelular, debe ser representativo de las alteraciones presentes en el tracto respiratorio. ${ }^{10}$

La citología convencional es una técnica de centrifugado durante diez minutos, 1.500-2.000 revoluciones por minuto, para luego extender el material obtenido sobre una lámina portaobjetos y colorearlo según la necesidad. ${ }^{10}$ El cytospin se realiza con un tipo especial de centrífuga utilizada para procesar muestras celulares para el análisis microscópico, la muestra se coloca en el delimitador de campo y se centrifuga a 1.000 revoluciones por minuto durante cinco minutos para ser coloreada. Esta última técnica está diseñada para concentrar células presentes en poca cantidad en un determinado fluido corporal. ${ }^{11}$

Aunque las dos técnicas identificaron el parásito, la procesada por cytospin redujo el tiempo de lectura, porque el diámetro del extendido fue menor, mientras que en la convencional el área del extendido fue mayor y requirió más tiempo y esfuerzo para realizar el diagnóstico. ${ }^{12}$

La presencia de estrongiloidiosis en países tropicales como Colombia es alta, por tanto debe tenerse en cuenta entre los diagnósticos diferenciales de cuadro clínicos compatibles, aún más si se considera que existe un alto porcentaje de casos no diagnosticados, subdiagnosticados o no reportados, lo cual obedece a tres razones principales: 1) los estudios de investigación sobre esta infección son escasos, haciendo que la información sobre su incidencia no sea clara ${ }^{1}$; 2) no suelen llevarse a cabo métodos diagnósticos de rutina para esta patología ${ }^{1}$; y 3 ) debido a que en la mayoría de la población la estrongiloidiosis suele seguir un curso subclínico puede existir un gran número de individuos inmunocompetentes infectados que permanecen 
sin diagnóstico ni tratamiento durante varios años. ${ }^{13}$ Cuando el uso de la terapia inmunosupresora comenzó a aumentar en regiones tropicales se elevó el riesgo de que el S. stercolaris pudiera causar serios problemas de salud pública. ${ }^{13}$ Es por ello que se hace necesario identificar la población en riesgo, aumentar la sospecha clínica y considerar el uso de técnicas como el cytospin y la citología convencional en diferentes muestras biológicas como el lavado broncoalveolar para realizar un diagnóstico oportuno.

\section{Referencias}

1. Schär F, Trostdorf U, Giardina F, Khieu V, Muth S, et. al. Strongyloides stercoralis: Global Distribution and Risk Factors. PLoS Negl Trop Dis. 2013 , 7(7):e2288

2. Fox LM. Strongyloidiasis. In: Centers for Disease Control and Prevention. Yellow book [monograph on the Internet]. Atlanta, GA: CDCP; 2014 [cited $2015 \mathrm{Ju}$ 13].Available from: http://wwwnc.cdc.gov/travel/yellowbook/2016/infectiousdiseases-related-to-travel/strongyloidiasis

3. Diaz L, Solano C, Rodriguez A. Strongiloidiasis diseminada posterior a Tratamiento de meningitis bacteriana. Univ. med. 2004; 45(1):32-6.

4. Fernández-Niño JA, Reyes-Harker P, Moncada-Álvarez LI, López MC, Chaves MP, Knudson A, et al. Tendencia y prevalencia de las Geohelmintiasis en La Virgen, Colombia 1995-2005. Rev. Salud pública. 2007; 9(2):289-96.
5. Buonfrate D, Requena-Mendez A, Angheben A, Muñoz J, Gobbi F, Van Den Ende J, et al. Severe strongyloidiasis: a systematic review of case reports. BMC Infect Dis. 2013 Feb 8;13:78.

6. Kassalik M, Mönkemüller K. Strongyloides stercoralis hyperinfection syndrome and disseminated disease. Gastroenterol Hepatol. 2011;7(11):766-8.

7. Mejia R, Nutman TB. Screening, prevention, and treatment for hyperinfection syndrome and disseminated infections caused by Strongyloides stercoralis. Curr Opin Infect Dis. 2012; 25(4):458-63.

8. Requena A, Chiodini P, Bisoffi Z, Buonfrate D, Gotuzzo E, Muñoz J. The laboratory diagnosis and follow up of strongyloidiasis: a systematic review. PLOS Negl Trop Dis. 2013. 7(1): e2002.

9. Arbeláez V, Angarita O, Gómez M, Martín A, Sprockel J, Mejía M. Presentación de caso clínico interinstitucional: gastroduodenitis severa secundaria a hiperinfección por strongyloides stercolaris en un hombre joven. Rev. Colomb. Gastroenterol. 2007. 22(2):118-25.

10. Mayayo E, Gomez-Aracil V, Azua-Blanco J, Capilla J, Mayayo R. Strongyloides stercolaris infection mimicking a malignant tumour in a non-immunocompromised patient. Diagnosis by bronchoalveolar cytology. J Clin Pathol. 2005; 58(4): 420-22.

11. Blenman Kim. Techniques in Cytology - Cytospin: Distinguishing Benign Cells from Malignant Cells. In: Sanguine Biosciences [monograph on the Internet]. Valencia, CA: 2013. Available from: http://technical.sanguinebio.com/techniquesin-cytology-cytospin-distinguishing-benign-cells-from-malignant-cells/

12. Ji-Youn S, Joungho H, Young Lyun O, Gee Young S, Kyeongman J, Taeeun $\mathrm{K}$. Value of bronchoalveolar lavage fluid cytology in the diagnosis of pneumocystis jirovecii pneumonia: a review of 30 cases. Tuberc Respir Dis. 2011; 71(5):322-27.

13. Olsen A, van Lieshout L, Marti H, Polderman T, Polman K, Steinmann P, et al Strongyloidiasis - - the most neglected of the neglected tropical diseases?. Trans R Soc Trop Med Hyg. 2009; 103(10): 967-72. 